Research Article

\title{
Robust Control of EHCS of Intelligent Commercial Vehicle under Load Change
}

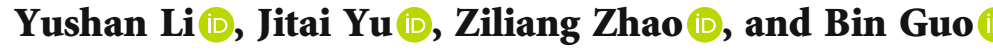 \\ Shandong University of Science and Technology, Qingdao, Shandong, China \\ Correspondence should be addressed to Ziliang Zhao; zhaoziliang1@sdust.edu.cn
}

Received 27 October 2021; Accepted 29 November 2021; Published 22 December 2021

Academic Editor: Chun-xi Yang

Copyright (C) 2021 Yushan Li et al. This is an open access article distributed under the Creative Commons Attribution License, which permits unrestricted use, distribution, and reproduction in any medium, provided the original work is properly cited.

\begin{abstract}
Electro Hydraulic Coupling Steering (EHCS) system is a new type of intelligent commercial vehicle steering system, having strong nonlinear characteristics. Besides, the change of load would cause the change of control system parameters, making not easy to establish an accurate control model of it. To realize the robustness of EHCS under the change of load, the controller based on the adaptive control method is proposed in this paper. To this end, the cosimulation model of EHCS is first established, where the constructed control model is simplified to a 2-degree-of-freedom model under reasonable simplification and assumption. Then, the steering angle controller is designed based on the model reference adaptive theory. Finally, some simulations are given to show the effectiveness of the proposed control method.
\end{abstract}

\section{Introduction}

As commercial vehicles pay more attention to use efficiency and cost savings, intelligenceof it is more urgent than that of passenger vehicles. In addition, there is large volume, high load, and higher center of mass in commercial vehicles and the demand for driving safety is stronger [1]. The steering system is an important part of intelligent commercial vehicle, which directly affects the handling, stability, and driving safety. The steering system of commercial vehicles has gradually developed from mechanical to intelligent and electric power [2], which has gone through several stages: hydraulic power (HPS), electronically controlled hydraulic power (ECHPS), electric power (EPS), and steer-by-wire (SBW), e.g. The traditional HPS requires a lot of energy and has the disadvantage that the assist characteristics can not be adjusted [3], which will make the driver feel heavy when turning at low speed and float at high speed. ECHPS realizes the adjustable assist characteristics on the basis of HPS. EPS can only be installed on light trucks although it has a good road feel [4]. SBW has no real mechanical feedback, and safety redundancy measures are not easy to ensure safety for commercial vehicles. The electrohydraulic coupled steering (EHCS) system in this paper adds a set of power motor unit on the basis of traditional HPS, which combines the advantages of high energy density of hydraulic system and high intelligence of electronic control system [5]. It can not only have good steering characteristics but also can be used as the hardware basis of active lateral control, matching the intelligent commercial vehicle.

EHCS is a complex nonlinear system, which has strong nonlinear characteristics such as hydraulic assistance and friction, e.g. [6]. Then, the load of the steering system will change with the vehicle speed, axle load, and other factors, and the parameters of the hydraulic system are time-varying, so it is not easy to establish an accurate control model [7-9]. Therefore, in order to control the angle of EHCS during autopilot, a simplified control model of EHCS must be established. Domestic and foreign scholars have done a lot of research on the steering system model. A 2-degree-of-freedom nonlinear hydraulic steering system model is proposed by Dell'Amico [10]. The system parameters are identified by the bench, and the model is linearized by the first-order Taylor expansion. Acarman et al. [8] approximate the hydraulic assist curve by using 8-degree polynomials and establish a 4degree-of-freedom model of an online hydraulic steering system. Tai and Tomizuka [11] established a 2-degree-offreedom linear model from motor torque to steering wheel 
angle, steering wheel angle to wheel angle by linearizing the assist curve, and identified the unknown parameters by an open-loop frequency test. Garcia et al. [12] also established the state equation model of the self-driving steering system of commercial vehicles by using the linearized assist curve and the equivalent model of the mechanical system with 2degree-of-freedom. Based on the above facts, the establishment of a 2-degree-of-freedom system model is sufficient for controller design.

As the low signal-to-noise ratio of the front wheel angle of the commercial vehicle, it can not be measured by the sensor, and the path tracking of the intelligent commercial vehicle is difficult to control the wheel directly. Therefore, the mainstream angle controller of EHCS uses the steering wheel angle instead of the wheel angle as the control target [13], and a large number of control methods have been proposed, such as fuzzy PID control [14], adaptive sliding control [15], and adaptive control $[16,17]$. The method of a nonlinear robust controller is proposed by Tai and Tomizuka [11]; a first-order filter is introduced to reduce the inherent jitter of a nonlinear robust controller. Acarman et al. [8] designed a sliding mode controller to satisfy the robust stability for the modeling boundary and parameter uncertainty of the linear hydraulic steering system. Based on the linearized 2-degree-of-freedom model of the EHCS, Garcia et al. [12] designed the steering angle PI controller. Some simple controllers such as the PI controller are not easy to adjust the change in EHCS while artificial intelligence control with complex structure needs long adjustment time, which can not achieve an instantaneous dynamic response. Adaptive control can automatically compensate for unpredictable changes such as model parameters and input signals [18] matching the EHCS, so the model reference adaptive theory is used to design the angle controller of EHCS.

From the above discussions, the controller of EHCS based on the adaptive control method is developed. The 2-degree-offreedom control model of EHCS is designed with reasonable assumptions and simplifies. Based on this model, the controller using model reference adaptive theory is designed by Lyapunov stability theory. Finally, the simulation results show that the system has good robustness under different load.

The main contributions of this paper are as follows. The cosimulation model and simplified 2-degree-of-freedom model are established for EHCS, which provides the basis for the development of the application of EHCS in the future. A controller based on model reference adaptive theory is proposed, which provides a new way to angle control of EHCS, and verifies the robustness of the controller under different load changes. It makes a certain contribution to the control of a steering system in an intelligent commercial vehicle.

The rest of the paper is organized as follows: Section 2 introduces the cosimulink model and simplified model. Section 3 designs a controller based on a parameter adaptive control system. Section 4 is the simulation results and analysis. Section 5 presents some conclusions.

\section{Modeling of EHCS}

The structure of EHCS is shown in Figure 1. The EHCS is divided into three parts: mechanical, hydraulic, and motor, in which the mechanical model includes a steering wheel, torsion, worm gear, and circulating ball power steering gear. Hydraulic module contacts of a rotating valve, hydraulic circuit, and hydraulic pump. The mechanical and hydraulic models are built in AMEsim, and the torque control model of permanent magnet synchronous motor (PMSM) is built in Simulink.

2.1. Establish Cosimulation Model. The mechanical model and hydraulic model are established in AMEsim; the mechanical part is partially simplified as follows. The damping coefficient between worm gears and worms is ignored. The intelligent commercial vehicle is driverless, and the driver input torque is zero. The torsional stiffness of the upper torsion bar is represented by the rotating spring damper. The equivalent moment of inertia between the steering string and the worm gear is represented by the upper rotating load, while the moment of inertia between the worm wheel and the lower torsion bar is represented by the lower rotating load. The lower torsion bar module is equivalent to a rotating spring with stiffness of $1.5 \mathrm{Nm} / \mathrm{deg}$, and the torsion bar deformation angle signal is collected by the steering sensor on the both ends of the spring. Motor driving torque is the only torque input of the model, which is built in Simulink. The circulating ball power steering gear is equivalent to a two-stage transmission pair containing a screw nut transmission pair and a rack tooth fan transmission pair [19]. The angle acquisition module is used to collect the rotation angle of the rocker shaft, ignoring the transmission ratio and elastic influence of the steering rod system. The rotation angle of the rocker shaft is regarded as the wheel angle.

The main component of the hydraulic module is the rotary valve, which is equivalent to a variable throttle according to its working principle [20], and the opening area of the variable throttle hole is controlled by the torsion bar deformation angle in the mechanical module. According to the parameters of the rotary valve and the method shown in the reference [19-21], the relationship between the overflow area of rotary valve port $A$ and the deformation angle of the torsion bar $\theta_{\mathrm{lg}}$ is calculated as shown in Equation (1). The hydraulic pump and piston adopt the module included in AMEsim.

$$
\begin{cases}7 A+85 \theta_{\mathrm{lg}}-180=0, & \theta_{\mathrm{lg}} \in[-5,-2], \\ 340 A+977 \theta_{\mathrm{lg}}-3486=0, & \theta_{\mathrm{lg}} \in(-2,1.4], \\ 8295 A-13083 \theta_{\mathrm{lg}}-69994=0, & \theta_{\mathrm{lg}} \in(1.4,5.35]\end{cases}
$$

where $A$ is the overflow area of the rotary valve and $\theta_{\lg }$ is the deformation angle of the torsion bar.

The cosimulation part with Simulink is established, in which the input is the steering resistance moment of the front axle and the motor torque and the output is the rotation angle of the two wheels, the required speed of the motor and the steering wheel angle. The final model is shown in Figure 2. 


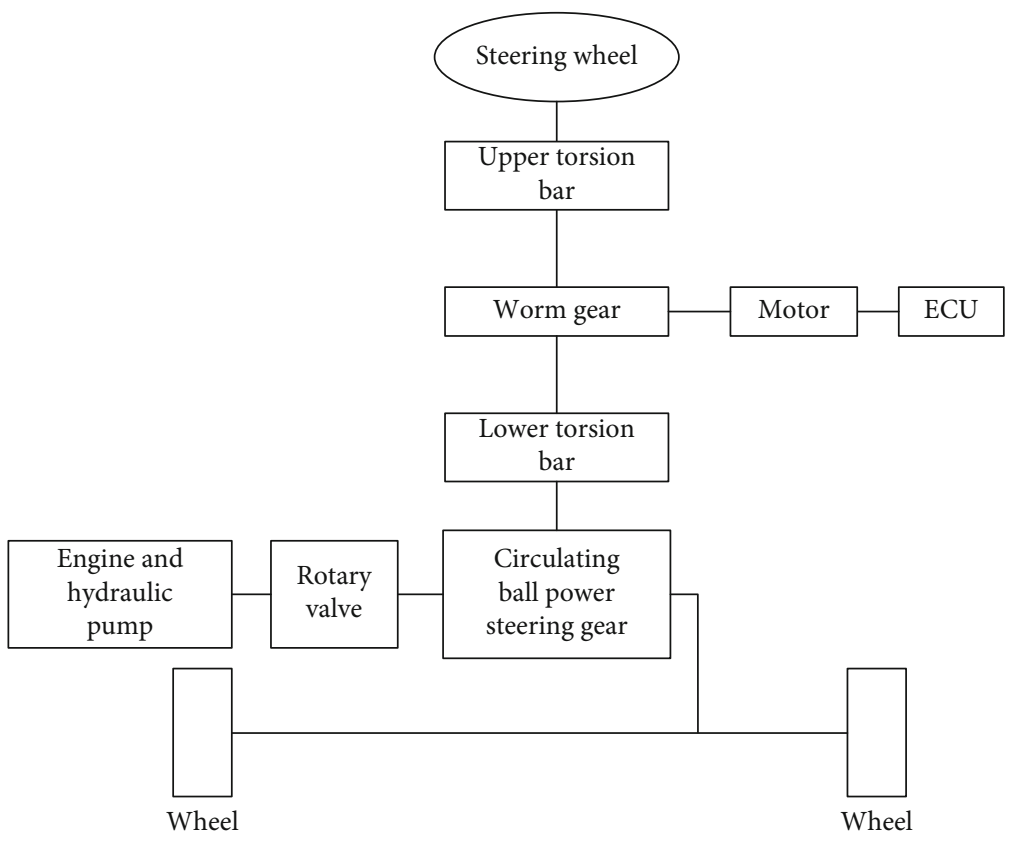

Figure 1: Structure diagram of EHCS.

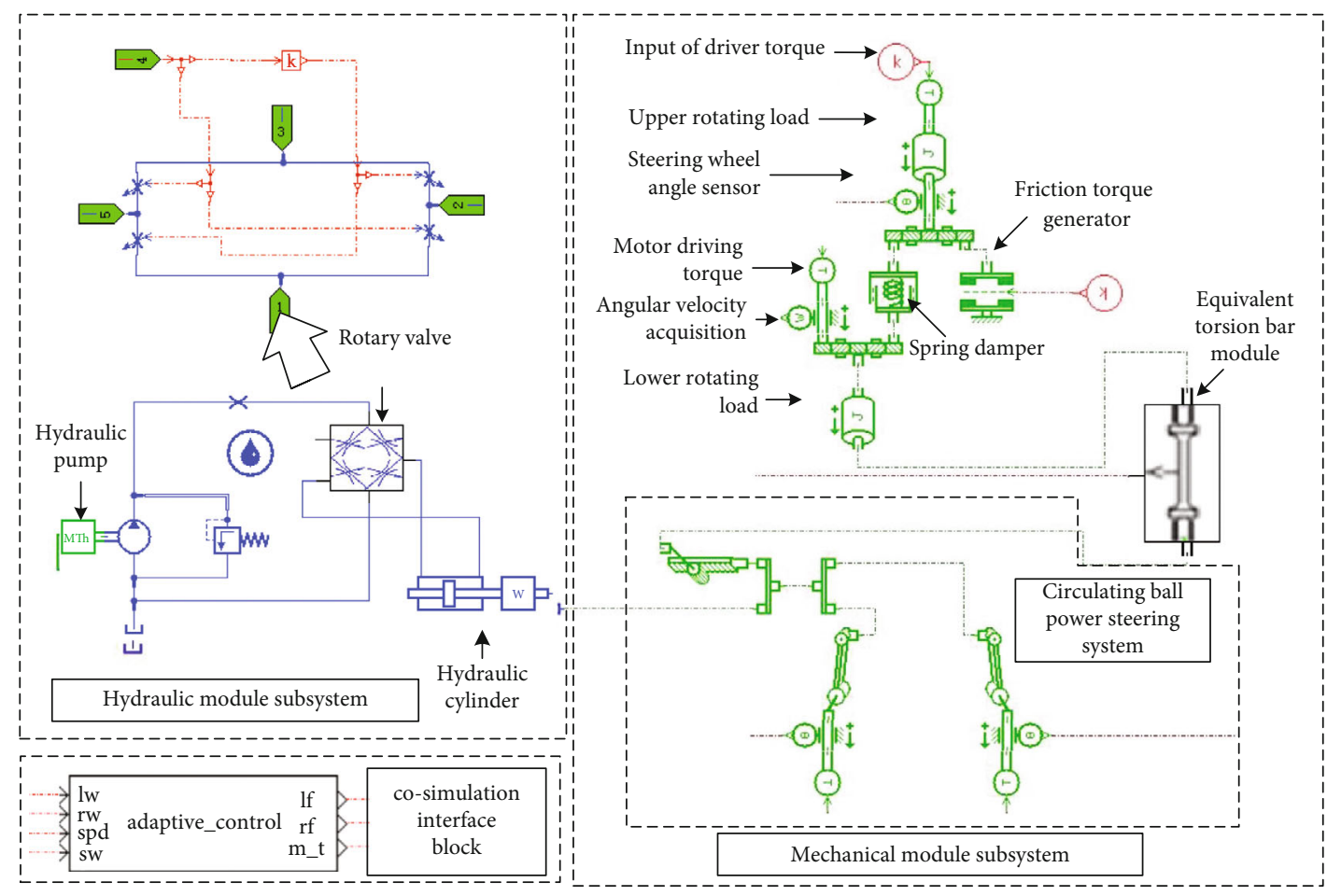

Figure 2: Mechanical and hydraulic system modeling in AMEsim.

The dynamic model of the permanent magnet synchronous motor is shown in Equation (2). In order to facilitate control, the strong coupling current and voltage should be decoupled. If the $d$-axis current is controlled to zero, the motor torque is proportional to the $q$-axis current. As shown in Equation (3), the motor torque can be controlled by controlling the $q$-axis current.

$$
\begin{gathered}
T_{e}=p_{n}\left[\psi_{f} i_{q}+\left(L_{d}-L_{q}\right) i_{q} i_{d}\right], \\
T_{e}=J_{e} \frac{d \omega_{m}}{d t}+R_{m} \omega_{m}+T_{L}, \\
T_{e 1}=p_{n} \psi_{f} i_{q},
\end{gathered}
$$




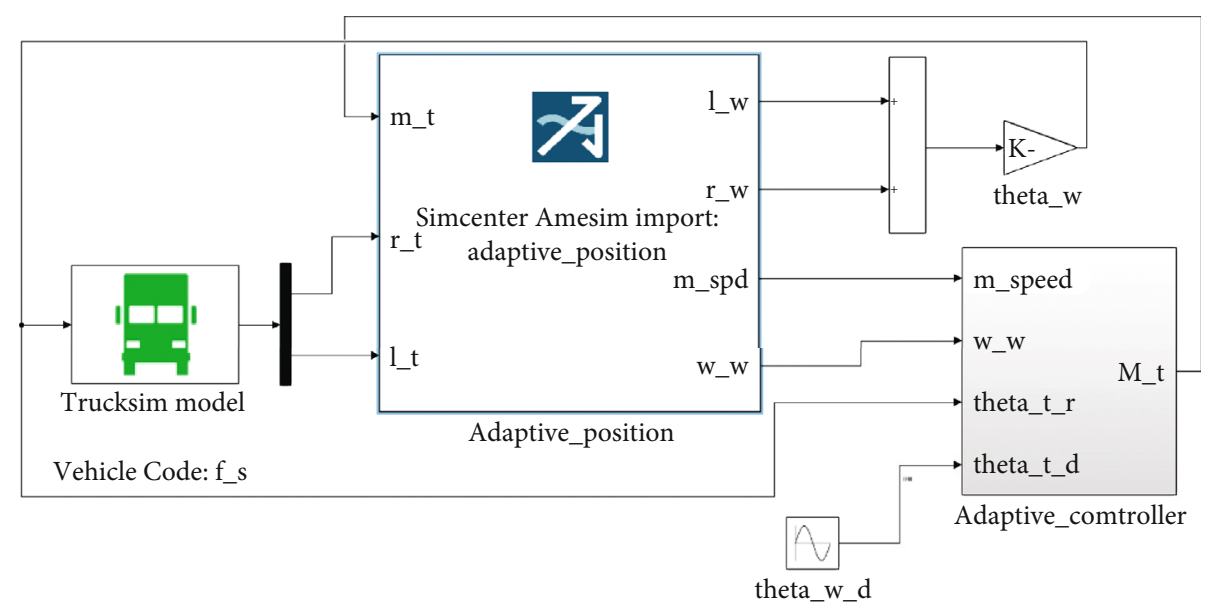

FIgURE 3: The combined simulation model.

where $T_{e}$ and $T_{e 1}$ are the motor electromagnetic torque, $T_{L}$ is the load torque, $\psi_{f}$ is the stator winding flux, $p_{n}$ is the motor pole logarithm, $L_{q}$ and $L_{d}$ are the $q$ - and $d$-axis inductance, respectively, $i_{q}$ and $i_{d}$ are the $q$ - and $d$-axis current, respectively, $J_{e}$ is the motor moment of inertia, $\omega_{m}$ is the rotor mechanical angular speed, and $R_{m}$ is the resistance coefficient.

The space vector pulse width modulation method, the inverter in Simulink, and the motor torque controller are used to build the torque control model of the permanent magnet synchronous motor (PMSM). The input of the model is the desired speed and $q$-axis current of the motor, and the output is the torque of the motor. In the TruckSim, a two-axle truck is selected, which is $5.5 \mathrm{t}$ at no load and $9.5 \mathrm{t}$ at full load. The transmission ratio of the steering system is set to 1 , and the flexibility is set to 0 . At this time, the wheel angle of the model in TruckSim is basically equal to the steering wheel angle. If the steering control of TruckSim is set to full, the wheel angle calculated in Simulink is input to the steering wheel angle in TruckSim, and the output of TruckSim is the steering resistance moment of the wheels on the front axle.

The cosimulation model is established as shown in Figure 3, including TruckSim function, AMEsim function, angle controller, and SVPWM motor controller.

2.2. Simplified Control Model. In order to simplify the EHCS as a 2-degree-of-freedom model, the following ignoring and assumptions are made: (1) inertia mass at the upper end of the lower torsion bar is equivalent to the worm gear, and inertia mass body at the lower end of the lower torsion bar is equivalent to the rocker shaft, dividing the whole steering system into two mass systems [20]; (2) the steering load is regarded as external interference; (3) the angle influence of the steering string is ignored; (4) the friction torque of the system is ignored; (5) the elastic influence of the steering rod system is ignored; (6) the elastic influence of the upper end of the upper torsion bar, that is, the steering string, is ignored. The force analysis of the simplified EHCS system is shown in Figure 4.
Ignoring the friction force of the system and linearizing the hydraulic assist force as $T_{h}=\psi_{0} T_{k 2}$, the dynamic model is shown in following:

$$
\begin{gathered}
J_{u} \ddot{\theta}_{u}+D_{u} \dot{\theta}_{u}+k_{2} \theta_{u}=T_{u}+n k_{2} \theta_{d}, \\
J_{d} \ddot{\theta}_{d}+D_{d} \dot{\theta}_{d}+\left(k_{r w}+n^{2} k_{2}+n \psi_{0} k_{2}\right) \theta_{d}=\left(n+\psi_{0}\right) k_{2} \theta_{u},
\end{gathered}
$$

where $J_{u}$ is the equivalent moment of inertia from the inertia mass at the top of the lower torsion bar to the worm gear, $\mathrm{kg} / \mathrm{m}^{2} ; D_{u}$ is upper mass damping coefficient, $\mathrm{Nm} / \mathrm{rad} /$ $s ; k_{2}$ is the elastic stiffness of upper torsion bar, $\mathrm{Nm} / \mathrm{rad} ; J_{d}$ is the equivalent moment of inertia from the bottom of the lower torsion bar to the tooth fan shaft, $\mathrm{kg} / \mathrm{m}^{2} ; D_{d}$ is lower mass damping coefficient, $\mathrm{Nm} / \mathrm{rad} / \mathrm{s} ; k_{r w}$ is the simplified from steering system load to the stiffness of the spring model, $\mathrm{Nm} / \mathrm{rad} ; T_{f}$ is friction torque of lower mass body, $\mathrm{Nm} ; n$ is the transmission ratio from the screw to the fan shaft, about 25; and $T_{h}$ is the equivalent moment of the hydraulic system acting on the lower mass body, Nm.

Based on the above assumptions and ignoring, the steering wheel angle is approximately the worm gear angle, which is given as

$$
\begin{aligned}
& \theta_{s}=\theta_{u}, \\
& \theta_{w}=\theta_{d},
\end{aligned}
$$

where $\theta_{s}$ is the angle of the steering wheel and $\theta_{w h}$ is the angle of the wheel.

The transfer function of the controlled system can be obtained as shown as

$$
G_{p}(S)=\frac{\theta_{w}(s)}{\theta_{s}(s)}=\frac{\theta_{d}(s)}{\theta_{u}(s)}=\frac{\left(n+\psi_{0}\right) k_{2}}{J_{d} s^{2}+D_{d} s+\left(k_{r w}+n^{2} k_{2}+n \psi_{0} k_{2}\right)} .
$$

After standardizing the transfer function, it is concluded that the transfer function is in the overdamping state, 


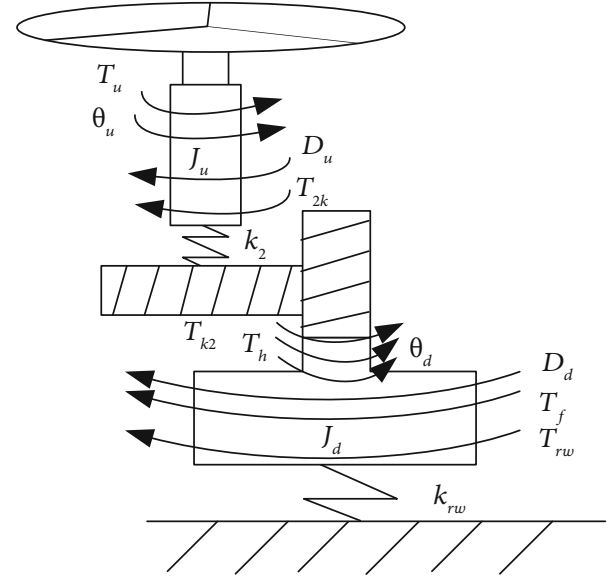

FIgURE 4: Diagram of force acting on a simplified system model.

meaning the response time is too long. In order to lay the groundwork for the model reference theory in the next chapter, an ideal transfer function model is proposed on the basis of the system transfer function. The following requirements are put forward for the ideal model: (1) It has a fast dynamic response and the rise time is less than 0.04 s. (2) The output amplitude should accurately express the input amplitude; in other words, the zero frequency amplitude should be 1. (3) The bandwidth of the system should meet the requirements of the steering system of the whole vehicle. (4) Within the required system bandwidth, the delay of the system should be less than 15 degrees as far as possible. The ideal model is a second-order model, and its transfer function is in a state of underdamping.

\section{Angle Control Strategy Based on Model Reference Adaptive Theory}

3.1. Design of Model Reference Adaptive Controller. Model reference adaptive control originates from the deterministic servo problem and is developed from the tracking problem. The system consists of a reference model, a controlled object, and a controller. The reference model is a given ideal model, which defines the performance indexes such as overshoot, damping performance, and adjustment time. The consistency between the controlled model and the reference model is measured by the state error vector [22]. The adaptive mechanism is designed according to the system stability criterion, and the corresponding adaptive law is designed to modify the variable parameters according to the direction of less deviation, so that the actual performance index of the system is close to the ideal performance index.

In this paper, aiming at the great variation of steering resistance moment with variable load of intelligent commercial vehicle and the nonlinear problems in the control of EHCS, a control framework is proposed based on model adaptive theory, and the control model is designed according to the ideal model as a reference model. Firstly, it is proposed that the state equation of the controlled system and the reference model with single input and single output is shown as

$$
\begin{gathered}
\dot{x}_{p}=A_{p} x_{p}+b_{p} u, \\
y_{p}=h^{T} x_{p}, \\
\dot{x}_{m}=A_{m} x_{m}+b_{m} u, \\
y_{m}=h^{T} x_{m},
\end{gathered}
$$

where $x_{p}$ is state vector of the controlled system, $u$ is controlled vector, $y_{p}$ is the output of a controlled system, $x_{m}$ is the state vector of the reference model, and $y_{m}$ is the output of the reference model.

The transfer functions of the controlled system and the reference model can be obtained from Equation (7) as shown as

$$
\begin{gathered}
G_{p}(s)=h^{T}\left(s I-A_{p}\right)^{-1} b_{p}=k_{p} \frac{N_{p}(s)}{D_{p}(s)}, \\
G_{m}(s)=h^{T}\left(s I-A_{m}\right)^{-1} b_{m}=k_{m} \frac{N_{m}(s)}{D_{m}(s)},
\end{gathered}
$$

where $k_{p}$ and $k_{m}$ are gain coefficients.

The angle control of the EHCS takes the steering wheel angle as the input and the rocker shaft angle as the output. Define the error between the reference model and the actual output of the controlled system as $e_{1}=y_{m}-y_{p}$, the control objectives of adaptive systems are shown as

$$
\lim _{t \longrightarrow \infty}\left|e_{1}\right|=\lim _{t \longrightarrow \infty}\left|y_{m}(t)-y_{p}(t)\right|=0 .
$$

In order to make the adaptive adjustable system match the reference model completely, the adaptive system should have enough variable parameters, and the number of variable parameters should be the same as the uncertain parameters of the system. Define four variable parameters $\sigma=\left[\begin{array}{llll}\sigma_{1} & \sigma_{2} & \sigma_{3} & \sigma_{4}\end{array}\right]$ and two auxiliary signals in order to design controller, the auxiliary signal is shown as

$$
\begin{aligned}
& \dot{\lambda}_{1}=A_{f} \lambda_{1}+b_{f} u, \\
& \dot{\lambda}_{2}=A_{f} \lambda_{2}+b_{f} y_{p},
\end{aligned}
$$

where $A_{f}$ and $b_{f}$ are related to the $N_{m}(s)$ of the transfer function of the reference model.

Define $w$ is the variable signal of the control system, $w$ $=\left[\begin{array}{llll}r & -\lambda_{1} & -y_{p} & -\lambda_{2}\end{array}\right]$, the controller is built as shown in Figure 5.

$\widehat{\sigma}$ is the variable parameter obtained in the ideal state, $\delta$ is the parameter error, and the augmented equation of state of the controlled system is obtained by substituting $\sigma=\widehat{\sigma}$ $+\delta$ into (7) as shown in (11). When $\delta=0$, the augmented equation of state of the system in the ideal state, that is, the augmented equation of state of the reference model, is obtained as shown as 


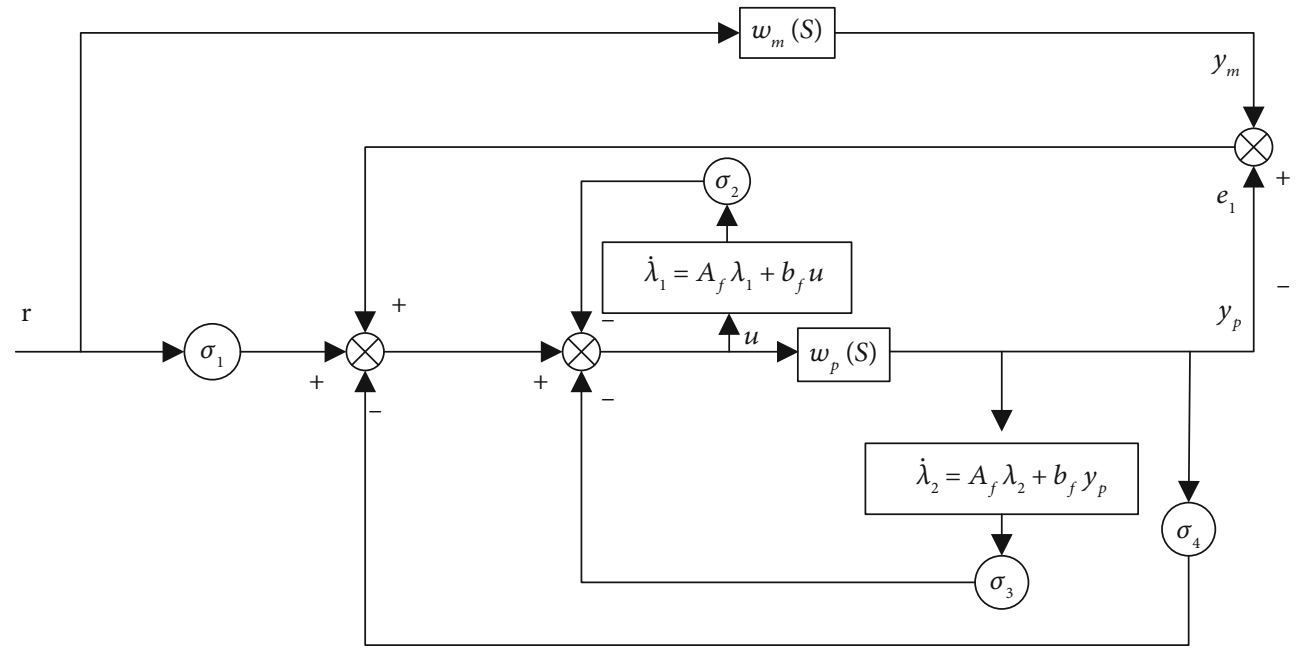

FIgURE 5: Control of model reference adaptive system.
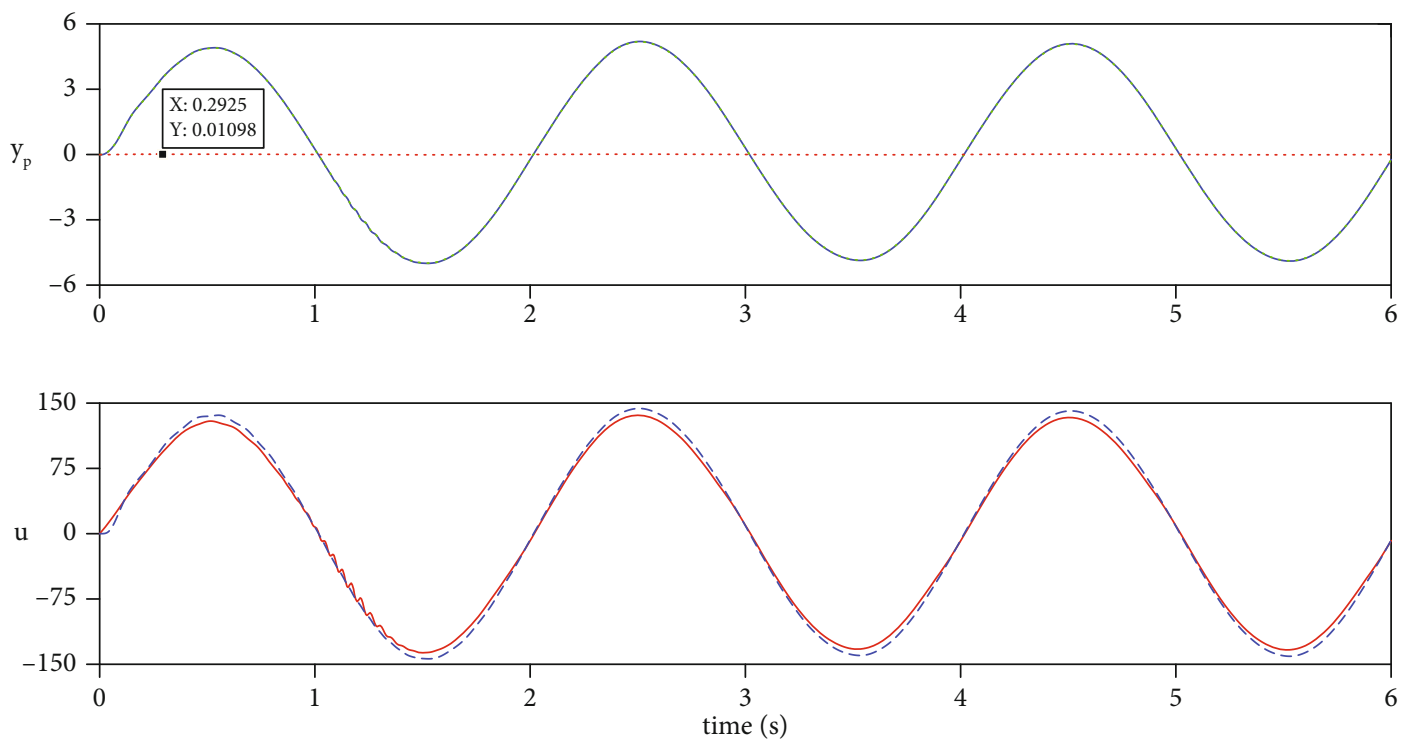

Figure 6: The control action $u$ and system output $y_{p}$.

$$
\begin{gathered}
\dot{x}=A_{c} x+b_{c}\left(\widehat{\sigma}_{1} r+\delta^{T} w\right), \\
\dot{x}_{m c}=A_{m c} x+b_{m c} \widehat{\sigma}_{1} r,
\end{gathered}
$$

where $x^{T}=\left[\begin{array}{lll}x_{p}^{T} & \lambda_{1}^{T} & \lambda_{2}^{T}\end{array}\right]$ and $x_{m c}{ }^{T}=\left[\begin{array}{lll}x_{m p}^{T} & \lambda_{m 1}^{T} & \lambda_{m 2}^{T}\end{array}\right]$. The augmented error state equation can be obtained by subtraction of Equation (11) as given as

$$
\dot{e}=A_{c} e+b_{c} \delta^{T} w=A_{c} e+b_{c} u_{c}
$$

where $e=\left[x_{p}-x_{m}, \lambda_{1}-\lambda_{m 1}, \lambda_{2}-\lambda_{m 2}\right]^{T}$ and $u_{c}=\delta^{T} w$.

3.2. Design of Adaptive Law according to Lyapunov Stability. The adaptive law is designed by using Lyapunov stability theory, which is defined according to the Lyapunov function to satisfy the stability of the system [23], and the Lyapunov function and its derivatives are defined as shown as

$$
\begin{aligned}
V & =\frac{1}{2}\left(e^{T} P e+\delta^{T} \Gamma^{-1} \delta\right) \\
\dot{V} & =\frac{1}{2} e^{T}\left(P A_{c}+A_{c}^{T} P\right) e+\delta^{T} w b_{c}^{T} P e++\delta^{T} \Gamma^{-1} \dot{\delta} \\
& =\frac{1}{2} e^{T}\left(P A_{c}+A_{c}^{T} P\right) e+\delta^{T}\left(w b_{c}^{T} P e+\Gamma^{-1} \dot{\delta}\right)=\dot{V}_{1}+\dot{V}_{2} .
\end{aligned}
$$

It is obvious that the function $V$ is positive definite, but in order to make the system stable, $\dot{V}$ should be negative definite.

According to Equation (12) and Kalman-Yakubovich theory [23], the transfer function $G_{e}(s)$ of $e$ is strictly positive real if and only if there are positive definite matrices $P$ and $Q$ , such that Equation (14) holds. Therefore, when the transfer function of $e$ is positive real, it can be obtained that $P A_{c}+$ 


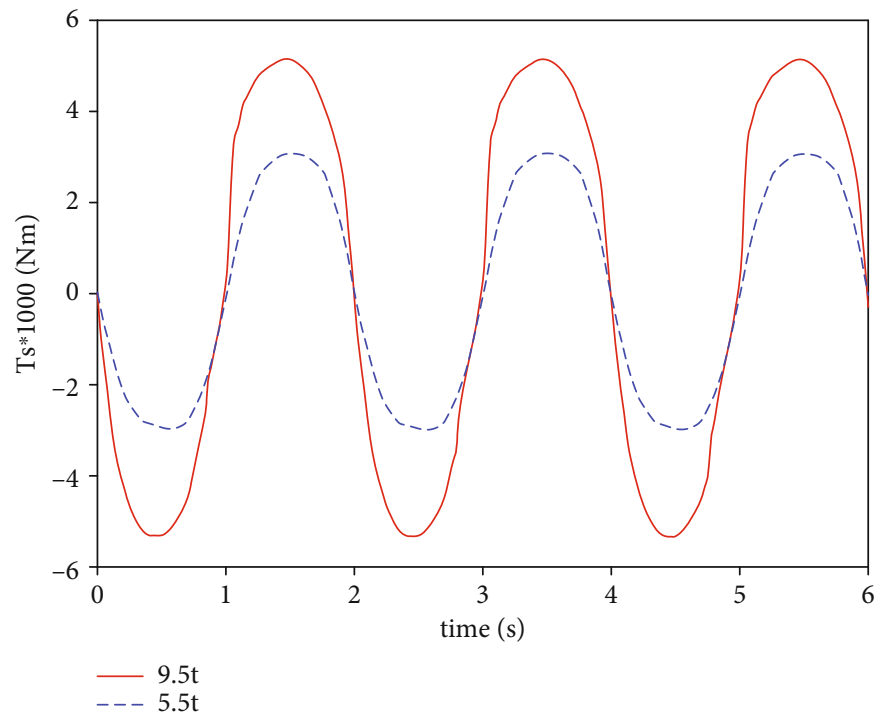

FIGURE 7: Steering resistance moment.
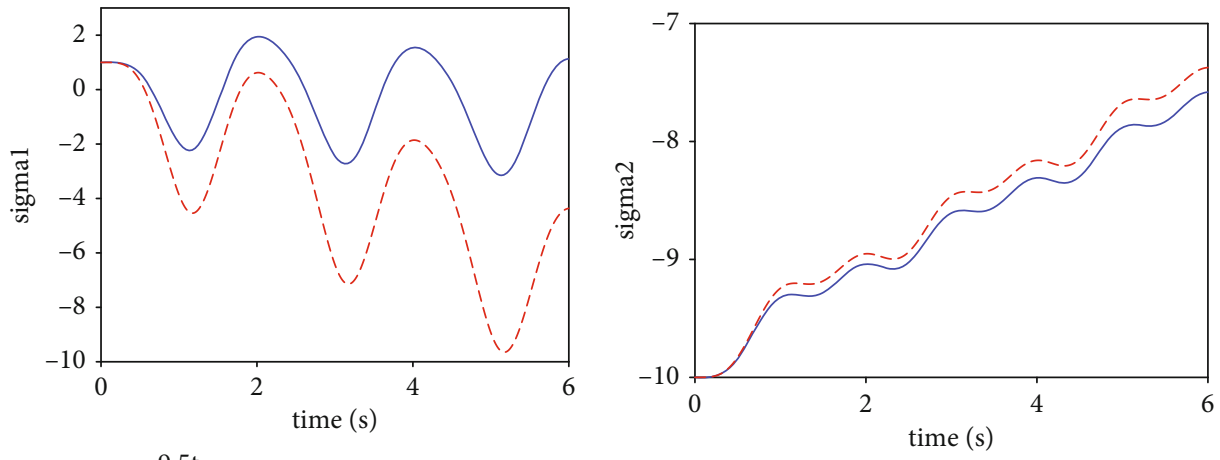

$-9.5 \mathrm{t}$
$---5.5 \mathrm{t}$

$-9.5 \mathrm{t}$
$---5.5 \mathrm{t}$

(a) Variable parameters $\sigma_{1}$

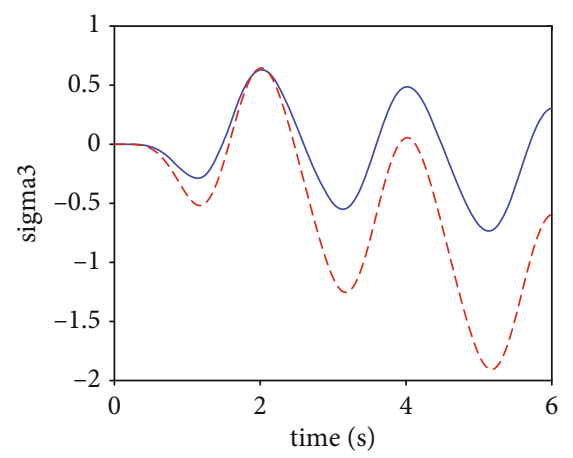

(b) Variable parameters $\sigma_{2}$

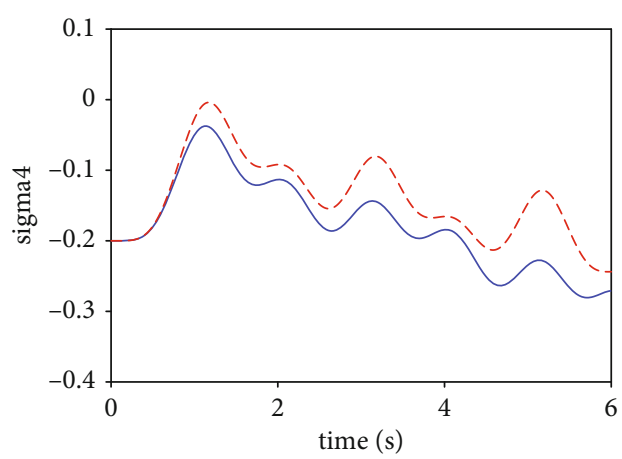

$-9.5 \mathrm{t}$
$---5.5 \mathrm{t}$

$-9.5 \mathrm{t}$

(c) Variable parameters $\sigma_{3}$

(b) Variable parameters $\sigma_{4}$

Figure 8: Comparison of variable parameters.

$A_{c}^{T} P=-Q$; by the words, $\dot{V}_{1}$ is negative definite.

$$
A^{T} P+P A=-Q .
$$

It is obviously to infer the $\dot{V}$ will be negative definite when $\dot{V}_{2}$ is zero. Hence, by defining adaptive law $\dot{\delta}=-\Gamma w$ $b_{c}^{T} P e, \dot{V}_{2}$ will be zero, which satisfies the condition that $\dot{V}$ is negative definite. 
In order to make the strictly positive realness of the error model, the auxiliary polynomials $L(s)=s+a$ are introduced, and the transfer functions of the system model, the reference model, and the error model are shown as

$$
\left\{\begin{array}{l}
w_{p}(S)=L(s) G_{p}(s), \\
w_{m}(S)=L(s) G_{m}(s), \\
w_{e}(S)=L(s) G_{e}(s) .
\end{array}\right.
$$

Define $\zeta=L^{-1}(S) w=\left[\begin{array}{llll}r & -\lambda_{1} & -y_{p} & -\lambda_{2}\end{array}\right]^{T} /(s+a)$, the adaptive law of system variable parameters designed according to Lyapunov stability theory is shown as

$$
\left\{\begin{array}{l}
\dot{\sigma}=\dot{\widehat{\sigma}}+\dot{\delta}=-\Gamma \zeta(t) e_{1} \\
\dot{\zeta}=-a \zeta(t)+w(t)
\end{array}\right.
$$

where $\Gamma$ is a positive definite symmetric matrix.

The input of the controlled system is shown as

$$
u=\sigma^{T} w(t)+e(t) \zeta^{T}(t) \Gamma \zeta(t)
$$

By introducing auxiliary polynomials $L(s)=s+2$, it can be obviously proved that $w_{p}(S), w_{m}(S)$, and $w_{e}(S)$ are strictly positive real. Define $\Gamma=\operatorname{diag}\left\{p_{i}\right\}, i=1,2,3,4$, according to the adaptive law of variable parameters as shown as

$$
\begin{gathered}
\dot{\sigma}=-\Gamma L^{-1}(S) w e_{1}(t), \\
\sigma=-\int_{0}^{t} \Gamma \zeta(t) e_{1}(t) d \tau+\sigma(0) .
\end{gathered}
$$

The input of the controlled object is shown as

$$
\begin{aligned}
u & =\sigma^{T}(t) w(t)+e_{1}(t) \zeta^{T}(t) \Gamma \zeta(t) \\
& =\left[-\int_{0}^{t} \sum p_{i} \zeta_{i} e_{1}(t) d \tau+\sigma(0)\right]^{T} w(t)+e_{1}(t) \sum p_{i} \zeta_{i}^{2}
\end{aligned}
$$

The $\sigma(0)$ is the initial value of variable parameters; it can be optional in theory; however, a bad initial value may cause the system not to work normally. In addition, the selection of weighting matrix $\Gamma$ has a great influence on the approximation reference model. Therefore, the selection of $\sigma(0)$ and $\Gamma$ should be careful.

\section{Robustness Verification of Angle Control Strategy}

The cosimulation model in Section 2 is used to track the desired rotation angle of the front wheel under the load of $9.5 \mathrm{t}$ and $5.5 \mathrm{t}$, respectively, and the sine waveform with a period of $2 \mathrm{~s}$ is selected to verify the simulation control strategy. The control action $u$ and system output $y_{p}$ under the two loads are shown in Figure 6, respectively. The steering resistance moment corresponding to the two loads is shown in Figure 7, and the change of variable parameters during the operation of the model is shown in Figure 8.

It can be seen from the above facts that given the same desired rotation angle, the maximum difference of steering resistance moment under different loads is about $3000 \mathrm{Nm}$, but under the same expected rotation angle, the actual turning angle after the control strategy is basically the same. There is a certain oscillation in the first $0.3 \mathrm{~s}$, which is caused by the same initial value of the variable parameters, and then, the system is stable after the variable parameters change with time. It is proved that the system has good robustness under the change of load.

\section{Conclusion}

Aiming at the EHCS with nonlinear uncertain parameters, after simplifying the control model to a 2-degree-of-freedom model, a controller based on adaptive theory is proposed by using Lyapunov stability theory. The adaptive law of variable parameters is designed, and the proposed control method can make the parameters change with time and compensate the nonlinear model disturbance. The load change is used to analyze the stability of control performance, and the results show that though the load difference is large, the operation of the EHCS can be effectively controlled and better performance can be obtained when tracking the target angle, meaning it has great robustness.

\section{Data Availability}

The data used to support the findings of this study are available from the corresponding author upon request.

\section{Conflicts of Interest}

The authors declare that there are no conflicts of interest regarding the publication of this paper.

\section{References}

[1] "Intelligent networked vehicle depth report: special topic on commercial vehicle applications," https://www.vzkoo.com/

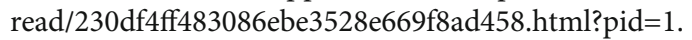

[2] G. Geng, H. Li, H. Jiang, J. Chen, and B. Tang, "Research on active return control of electro-hydraulic coupling steering system of commercial vehicle," Automotive Engineering, vol. 43, no. 6, pp. 899-908, 2021.

[3] Z. Tang, Research on Path Tracking Control of ElectroHydraulic Coupling Steering System for Commercial Vehicles [Master], Chongqing Jiaotong University, China, 2019.

[4] J. Sun, Y. Zhang, X. Shi, and J. Li, "Summary of the development of automobile power steering technology," Heavy truck, vol. 4, pp. 13-14, 2011.

[5] W. Cao, Study on Characteristics and Lateral Motion Control of Electro-Hydraulic Coupled Power Steering System for Commercial Vehicles[Master], Yanshan University, China, 2020.

[6] K. Yu, "Development of electronically controlled hydraulic power steering system for NJ1062 freight car," Journal of Nanjing Institute of Engineering, vol. 1, no. 12, pp. 71-74, 2014. 
[7] S. Wang, G. Shi, H. Zhang, C. Ju, and D. Sang, "Auto disturbance rejection control of steering wheel torque of electrohydraulic coupling steering system," Automotive Engineering, vol. 43, no. 5, pp. 770-775+790, 2021.

[8] T. Acarman, K. A. Redmill, and U. Ozguner, "A robust controller design for drive by wire-hydraulic power steering system," in Proceedings of the 2002 American Control Conference, Hilton Anchorage and Egan Convention Center, Anchorage, Alaska, USA, 2002.

[9] C. Lv, Research on Control Strategy of Electro-Hydraulic Coupling Steering System of Commercial Vehicle[Master], Jilin University, China, 2017.

[10] A. Dell'Amico, On Electrohydraulic Pressure Control for Power Steering Applications, 67 Linköping University, Sweden, 2016.

[11] M. Tai and M. Tomizuka, "Nonlinear robust loop shaping controller design for automated lane guidance of heavy vehicles," in Proceedings of the American Control Conference, pp. 2028-2032, Chicago, IL, USA, 2000.

[12] O. Garcia, J. V. Ferreira, and A. de Miranda, "Dynamic model of a commercial vehicle for steering control and state estimation," in XI Simposio Brasileiro de Automatice Inteligente 69 (SBAI), Chicago, IL, USA, 2013.

[13] W. Zhao, K. Gao, and W. Wang, "Prevention of instability control of commercial vehicle based on electric-hydraulic coupling steering system," Journal of Jilin University (Engineering and Technology Edition), vol. 48, no. 5, pp. 1305-1312, 2018.

[14] J. Liu, Y. Liang, J. Chen, S. Wang, and B. Yan, "Research on brushless DC motor control system based on fuzzy PID control," Journal of Physics: Conference Series, vol. 1, no. 2033, pp. 1742-1750, 2021.

[15] C. Guan and S. Pan, "Adaptive sliding mode control of electrohydraulic system with nonlinear unknown parameters," Control Theory \& Applications, vol. 16, no. 11, pp. 1275-1284, 2008.

[16] Y. Li, R. Zhou, Q. Zheng, and L. Yang, "Design of model reference adaptive control law of steering mechanism for heavy duty vehicle," in IEEE International Conference on Industrial Informatics, pp. 347-352, Beijing, China, 2012.

[17] J. Zhao, Y. Lv, and Z. Zhao, "Adaptive learning based outputfeedback optimal control of CT two-player zero-sum games," in IEEE Transactions on Circuits and Systems II: Express Briefs, 2021.

[18] W. Liu, L. Chen, and J. Chen, "Adaptive internal model control for automotive steer-by-wire system with time delay," Journal of Shanghai Jiao Tong University, vol. 55, no. 428, pp. 12101218, 2021.

[19] P. Pfeffer, M. Harrer, and D. Johnston, "Modelling of a hydraulic steering system," Fisita World Automotive Congress, 2006.

[20] Y. Chi, Research on Lateral Control for Path Tracking of Commercial Vehicle Based on Electro-Hydraulic Coupled Steering[Master], Shandong University of Science and Technology, China, 2019.

[21] M. Tai, P. Hingwe, and M. Tomizuka, "Modeling and control of steering system of heavy vehicles for automated highway systems," IEEE/ASME Transactions on Mechatronics, vol. 9, no. 4, pp. 609-618, 2004.

[22] Y. Li and K. Zhang, Adaptive Control Theory and Application, Northwest University of Technology Press, Xi'an, China, 2005.
[23] H. Liao, C. Huang, and Y. Huang, "Control of AC servo motor based on Narendra stable adaptive controller," Mechanical research and application, vol. 27, no. 6, pp. 175-178, 2014.

[24] J. Zhao, J. Na, and G. Gao, "Adaptive dynamic programming based robust control of nonlinear systems with unmatched uncertainties," Neurocomputing, vol. 395, pp. 56-65, 2020. 